\title{
Steiner Minimal Trees on Sets of Four Points
}

\author{
D. Z. Du, ${ }^{1}$ F. K. Hwang, ${ }^{2}$ G. D. Song, ${ }^{3}$ and G. Y. Ting ${ }^{4}$ \\ ${ }^{1}$ University of California, Santa Barbara, California, USA, also from Academia Sinica, Bejing, China \\ ${ }^{2}$ AT\&T Bell Laboratories, Murray Hill, NJ 07974, USA \\ ${ }^{3}$ Quiquihaer Light Engineering College, Heilungiang, China \\ ${ }^{4}$ Quiquihaer Teachers' College, Heilungjiang, China
}

\begin{abstract}
Let $S=\{A, B, C, D\}$ consist of the four corner points of a convex quadrilateral where diagonals $[A, C]$ and $[B, D]$ intersect at the point $O$. There are two possible full Steiner trees for $S$, the $A B-C D$ tree has $A$ and $B$ adjacent to one Steiner point, and $C$ and $D$ to another; the $A D-B C$ tree has $A$ and $D$ adjacent to one Steiner point, and $B$ and $C$ to another. Pollak proved that if both full Steiner trees exist, then the $A B-C D(A D-B C)$ tree is the Steiner minimal tree if $X A O D>$ $(<) 90^{\circ}$, and both are Steiner minimal trees if $\triangle A O D=90^{\circ}$. While the theorem has been crucially used in obtaining results on Steiner minimal trees in general, its applicability is sometimes restricted because of the condition that both full Steiner trees must exist. In this paper we remove this obstacle by showing: (i) Necessary and sufficient conditions for the existence of either full Steiner tree for $S$. (ii) If $\angle A O D \geq 90^{\circ}$, then the $A B-C D$ tree is the SMT even if the $A D-B C$ tree does not exist. (iii) If $\Varangle A O D<90^{\circ}$ but the $A D-B C$ tree does not exist, then the $A B-C D$ tree cannot be ruled out as a Steiner minimal tree, though under certain broad conditions it can.
\end{abstract}

\section{Introduction}

A Steiner minimal tree (SMT) for a given set $P$ of points in the Euclidean plane is the shortest tree interconnecting $P$. Any intersections of edges which are not in $P$ are called Steiner points. It is well known [5] that each Steiner point is of degree three and any two edges in an SMT intersect at an angle with at least $120^{\circ}$. An interconnecting tree satisfying the above two conditions is called a Steiner tree. It is also well known [5] that a Steiner tree for $n$ given points can have at most $n-2$ Steiner points. A Steiner tree is full if it has $n-2$ Steiner points. 
Melzak [6] gave an elegant method of constructing a Steiner tree with a given topology. However, the construction of an SMT for a general set $P$ is known [4] to be an $N P$-complete problem, largely due to the exploding number of possible topologies. Therefore, results which can rule out certain topolgies by using some simple properties of the given set $P$ or subsets of $P$ would be very helpful. Currently, the interconnection of three points is very well understood. We have the following necessary and sufficient conditions to determine an SMT: if the triangle formed by the three points contains no angle of $120^{\circ}$ or more, then the shortest connection for these three points is the unique full Steiner tree, otherwise the shortest connection consists of the two shorter sides of the triangle. However, the interconnection of four points is much more complicated and our current knowledge is quite incomplete. We quote the following four results from the literature.

Let $S=\{A, B, C, D\}$ denote the given set of four points and denote the two full Steiner trees as shown in Fig. 1(a) and (b) by the $A D-B C$ tree and the $A B-C D$ trees, respectively.

The first three results are due to Pollak [8].

Theorem 1. The existence of either full Steiner tree implies that ABCD is a convex quadrilateral.

Throughout this paper we assume that the two diagonals $[A, C]$ and $[B, D]$ intersect at $O$.

Theorem 2. Suppose that both full Steiner trees for $S$ exist. Then

(length of the $A D-B C$ tree) (length of the $A B-C D$ tree)

if and only if $\triangle A O D \equiv 90^{\circ}$.

We call the $A D-B C$ tree the acute (obtuse) full Steiner tree if $\Varangle A O D \leq(\geq) 90^{\circ}$.

Theorem 3. Suppose that both full Steiner trees for $S$ exist. Then the acute one is the SMT for $S$ and both are if $X A O D=90^{\circ}$.

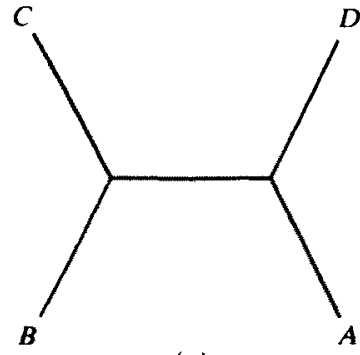

(a)

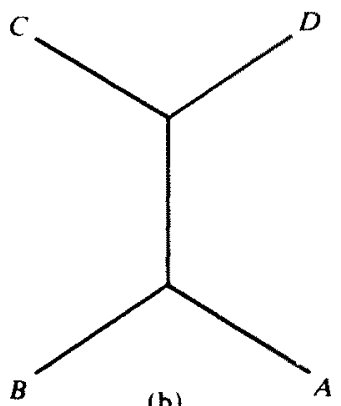

(b)

Fig. 1. Two full Steiner trees for four points. 
Ollerenshaw [7] proved

Theorem 4. Suppose that both full Steiner trees for $S$ exist. Then the shorter tree is always the one with the longer center edge (edge connecting the two Steiner points).

She also gave credit to Sir Bondi for proving Theorem 2 for the case $\Varangle A O D=$ $90^{\circ}$. While Theorem 4 gives an interesting property for SMT, it does not help in ruling out the longer full Steiner tree from consideration since one has to construct it first. On the other hand, Theorem 3 has been crucially used in finding SMTs for special point-sets or in determining their properties [1]-[3], [8]. However, even the conditions of Theorem 3 are sometimes difficult to apply. In this paper we attempt to promote the applicability of Theorem 3 by answering the following three questions:

1. What are the necessary and sufficient conditions for the existence of a full Steiner tree for $S$ ?

2. Suppose that the acute full Steiner tree exists. Is it the SMT regardless of the existence of the obtuse full Steiner tree?

3. Suppose that the obtuse full Steiner tree exists. Is it never the SMT regardless of the existence of the acute full Steiner tree?

We give complete answers to all three questions.

\section{Some Preliminary Results}

The notation $\Varangle X Y Z$ means the angle extending from line $[X, Y]$ counterclockwise to line $[Y, Z]$. For two given points $X$ and $Y$ the notation $(X Y)$ denote the point $Z$ such that $X Y Z$ is an equilateral triangle and $\measuredangle Y X Z=60^{\circ}$; the notation $d[X, Y]$ denote the distance between $X$ and $Y$.

Let $A B C$ be a triangle. Define $E=(A(B C))$ and $F=((A B) C)$. Construct equilateral triangles $\triangle A B(A B), \triangle B C(B C), \triangle A(B C) E$, and $\triangle C(A B) F$ (Fig. 2).

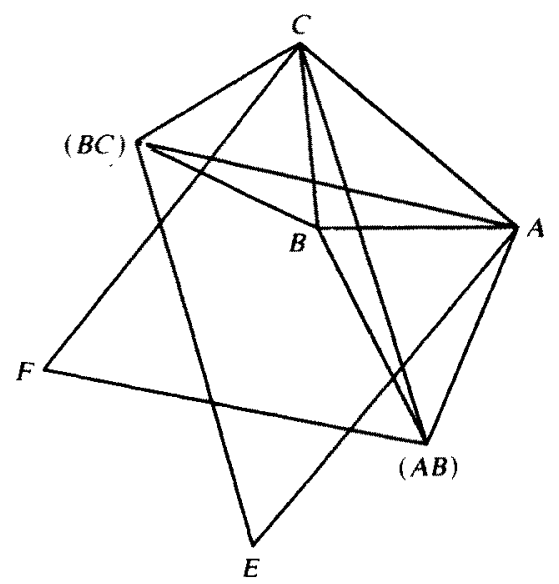

Fig. 2. A triangle and some equilateral triangles. 
Lemma 1. (i) $B F E$ is an equilateral triangle.

(ii) $A C F E$ is a parallelogram.

Proof. Since $\triangle A B(B C) \equiv \triangle(A B) B C$, we have $d[A,(B C)]=d[C,(A B)]$. Since $\triangle(A B) A E \equiv \triangle B A(B C)$, we have $d[(A B), E]=d[B,(B C)]$. Similarly we have $d[(B C), F]=d[B,(A B)]$. It is now easy to show that $\triangle(A B) A E \equiv \triangle B A(B C) \equiv$ $\triangle B(A B) C \equiv \triangle(B C) F C$. In particular $d[A, E]=d[C, F]$. Furthermore,

$$
\begin{aligned}
\Varangle F(A B) E & =180^{\circ}-\Varangle(A B) E A-\measuredangle E A(A B)-\{120-\Varangle C(A B) B\} \\
& =60^{\circ}-\Varangle(B C) C F=\Varangle F C B \\
& =60^{\circ}-\Varangle B(B C) A=\Varangle A(B C) C=\Varangle E(B C) B .
\end{aligned}
$$

Therefore $\quad \triangle F(A B) E \equiv \triangle F C B \equiv \triangle A(B C) C \equiv \triangle E(B C) B \quad$ and $\quad d[E, F]=$ $d[B, F]=d[A, C]=d[B, E]$. We have shown that $A C F E$ is a parallelogram since opposite sides are equal.

Lemma 2. Let $S=\{A, B, C, D\}$ where the four points form a convex quadrilateral. Let $E=(A(B C))$ and $F=((A B) C)$ as before (see Fig. 3). Then

$$
d[(D A),(B C)]=d[D, E] \gtreqless d[D, F]=d[(A B),(C D)] \quad \text { if } \Varangle A O D \geqq 90^{\circ} \text {. }
$$

Proof. It is easily verified that $\triangle(D A) A(B C) \equiv \triangle D A E$ and $\triangle(C D) C(A B) \equiv$ $\triangle D C F$. Hence $d[(D A),(B C)]=d[D, E]$ and $d[(A B),(C D)]=d[D, F]$. Furthermore, since $\triangle B(A B) E \equiv \triangle A B C$, we have $\Varangle C A B=\Varangle E B(A B)$. It follows that $\triangle E B D=\measuredangle A O D+60^{\circ}$ and $\measuredangle D B F=300^{\circ}-\measuredangle E B D=240^{\circ}-\Varangle A O D$. Compare $\triangle D B E$ and $\triangle D B F$. If $\angle E B D \geq 180^{\circ}$, then $d[D, E]>d[B, F]$ by the law of cosines since $\triangle D B E>\triangle D B F$. If $\Varangle E B D<180^{\circ}$, then, again by the law of cosines,

$$
d[D, E] \gtreqless d[D, F] \quad \text { if } \quad \measuredangle E B D \gtreqless \triangle D B F
$$

or, equivalently, if $\triangle A O D \gtreqless 90^{\circ}$.

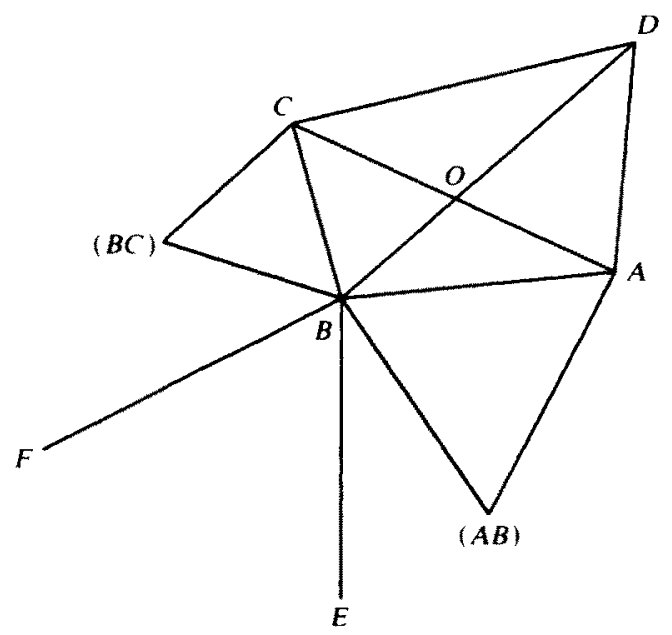

Fig. 3. Comparison of $d[D, E]$ and $d[D, F]$. 
Note that $d[D, E]$ is the length of the $A D-B C$ tree if it exists and $d[D, F]$ is the length of the $A B-C D$ tree if it exists. Therefore Theorem 1 follows from Lemma 2 as a corollary.

We will call $[(A B),(C D)]$ or $[D, F]$ an axis of the $A B-C D$ tree. Note that there exist other axes of the $A B-C D$ tree analogous to $[D, F]$. Lemma 2 says that all of them are equal. Of course the same is true for all axes of the $A D-B C$ tree. We now answer question 2 in the affirmative.

Theorem 5. The acute full Steiner tree, if existed, is the SMT.

Proof. Pollak proved Theorem 3 by showing that any spanning tree is longer than either an axis of the $A B-C D$ tree or an axis of the $A D-B C$ tree regardless of the existence of the two trees. However, the relations between these axes, for example, Theorem 2, do depend on the existence of the two trees. Lemma 2 provides these relations when the existence is not assumed.

We now give three lemmas to be used later. Lemma 3 was first noted by Ollerenshaw [7].

Lemma 3. Suppose that $\Varangle C A B=120^{\circ}$. Then $\Varangle(B C) A B=60^{\circ}$ (see Fig. 4).

Proof. The four points $A, B,(B C)$, and $C$ are cocircular. Hence $\Varangle(B C) A B=$ $\Varangle C A(B C)=60^{\circ}$ by noting that $\Varangle B C(B C)$ is equilateral.

Lemma 4. Let $A B C D$ be a quadrilateral with $\Varangle A+\Varangle C \geq 180^{\circ}$ (see Fig. 5). Then $\Varangle C A B \geq \Varangle C D B$ (equality is attained only when $\Varangle A+\Varangle C=180^{\circ}$ ).

Proof. $\Varangle A+\not C \geq 180^{\circ}$ implies that $A$ is on or inside of the circle circumscribing $\triangle B C D$. Hence $\Varangle C A B \geq \Varangle C D B$.

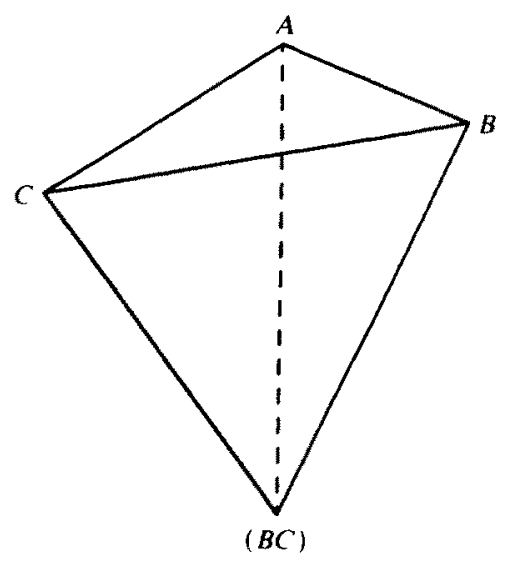

Fig. 4. $[A,(B C)]$ divides $\Varangle A$. 

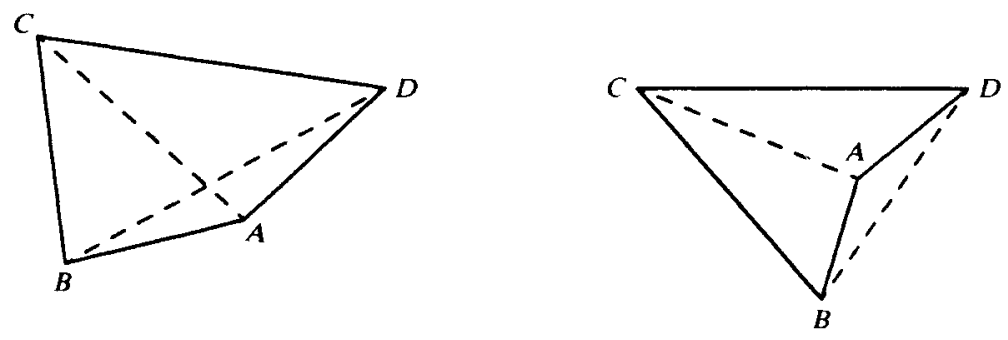

Fig. 5. $\Varangle C A B \geqslant \Varangle C D B$.

Let $T_{i}, i \in\{A, B, C, D\}$, denote the Steiner tree for $\{A, B, C, D\}$ with exactly one Steiner point and that Steiner point is not adjacent to $i$.

Lemma 5. Suppose that in the quadrilateral $A B C D \Varangle A \geq 120^{\circ}$ and $\Varangle B \geq 120^{\circ}$. Then the length of any Steiner tree for $\{A, B, C, D\}$ with a single Steiner point is longer than $d[(A B),(C D)]$.

Proof. It is easily shown that $T_{C}$ and $T_{D}$ do not exist. $T_{B}$ has length $d[A,(C D)]+$ $d[A,(A B)]$ and $T_{A}$ has length $d[B,(C D)]+d[B,(A B)]$. Both are greater than $d[(A B),(C D)]$ by triangle inequality.

\section{The Existence of a Full Steiner Tree}

Theorem 6. Necessary and sufficient conditions for the existence of the $A D-B C$ tree (Fig. 6) are:

(i) The quadrilateral $A B C D$ is convex.

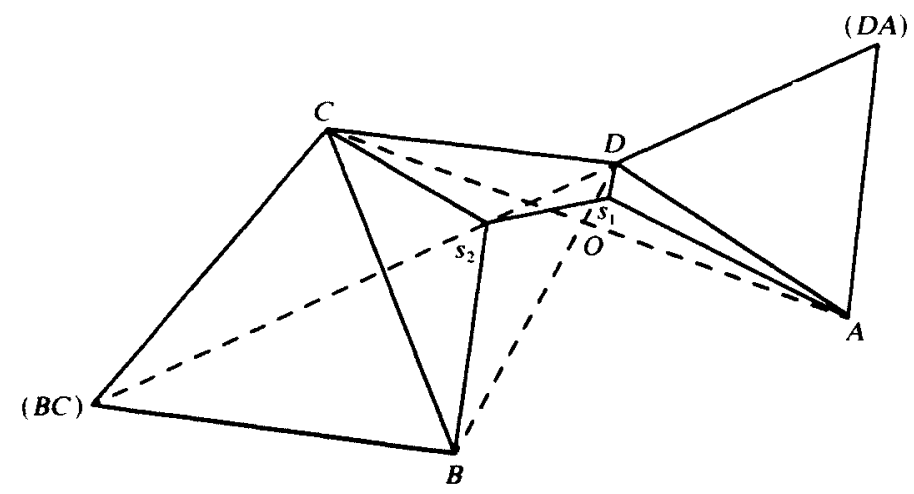

Fig. 6. The $A D-B C$ tree. 
(ii) $\Varangle D A(B C), \Varangle(B C) D A, \Varangle(A D) B C$, and $\Varangle B C(A D)$ are all less than $120^{\circ}$. (iii) $\measuredangle A O D<120^{\circ}$.

Proof. The necessity of (i) follows from Theorem 1. Assuming condition (i) is satisfied, we show that conditions (ii) is necessary and sufficient for $[(D A),(B C)]$ to lie inside the polygon $(D A) A B(B C) C D$. It is easily seen that $[(D A),(B C)]$ lies below $[(D A), D]$ if and only if $\Varangle(B C) D A<120^{\circ}$. Similar statements can be made for the other three angles. But $[(D A),(B C)]$ lying below $[(D A), D]$ and $[(B C), C]$, and lying above $[(D A), A]$ and $[(D A), B]$ implies that $[(D A),(B C)]$ lies inside the polygon $(D A) A B(B C) C D$.

Finally we show that if $A B C D$ satisfies (i) and (ii), then the $A D-B C$ tree exists if and only if $\triangle A O D<120^{\circ}$. Suppose that the $A D-B C$ tree exists. Since $\left[D, s_{1}\right]$ is parallel to $\left[B, s_{2}\right], s_{1}$ and $s_{2}$ must lie on different sides of $[D, B]$. Similarly, they must lie on differeent sides of $[A, C]$. In other words, $s_{1}$ lies within $\triangle A O D$ and $s_{2}$ lies within $\triangle B O C$. Hence $\triangle A O D<\triangle A s_{1} D=120^{\circ}$.

Next suppose that $\Varangle A O D<120^{\circ}$. Without loss of generality assume that $O$ lies below $[(D A),(B C)]$ and let $[(D A),(B C)]$ cross $[D, O]$ and $[C, O]$ at $U$ and $V$, respectively (see Fig. 7). We will show that either $\Varangle A U D \leq 120^{\circ}$ or $\Varangle C V B<$ $120^{\circ}$. Suppose, say, $\Varangle C V B<120^{\circ}$. Then the circle circumscribing $\triangle B C(B C)$ intersects $[(D A),(B C)]$ at a point to the left of $V$. Since $\measuredangle A V D<\triangle A O D<120^{\circ}$, the circle circumscribing $\triangle A D(D A)$ intersects $[(D A),(B C)]$ at a point to the right of $V$. By Melzak's construction the $A D-B C$ tree exists.

Let $Y$ be a point on $[O, D]$ such that $\measuredangle A Y D=120^{\circ}$ and let $X$ be a point on $[O, C]$ such that $\Varangle C X B=120^{\circ}$. Connect $(D A)$ and $Y, Y$ and $X, X$ and $(B C)$ (see Fig. 8). By Lemma $3 \not(D A) Y D=\Varangle C X(B C)=60^{\circ}$. Hence

$$
\begin{aligned}
\not(B C) X Y+\not X Y Y(D A) & =(B C) X O+(\not O X Y+\not X Y O)+\not O Y(D A) \\
& <120^{\circ}+120^{\circ}+120^{\circ}=360^{\circ} .
\end{aligned}
$$

It follows at least one of the points $X$ and $Y$ lies above $[(D A),(B C)]$. Without loss of generality assume it is $X$. Then ${ }_{4} C V B<\Varangle C X(B)=120^{\circ}$. The proof is complete.

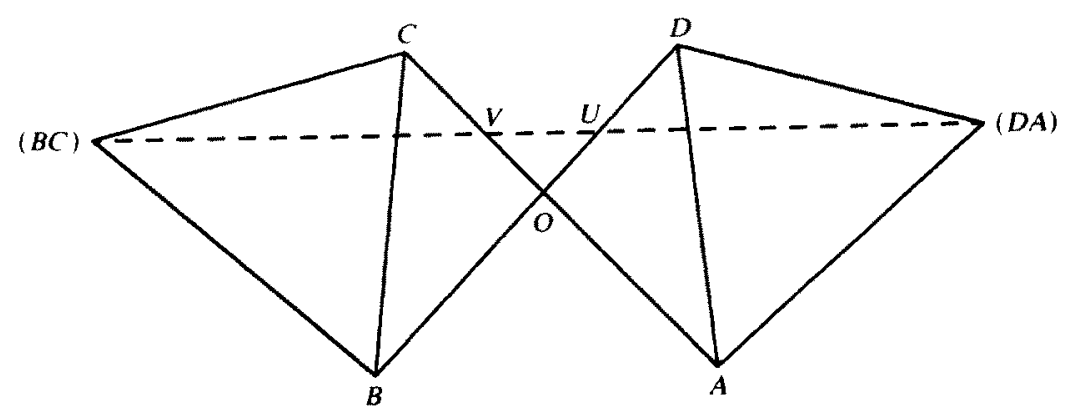

Fig. 7. $V$ is to the left of $U$. 


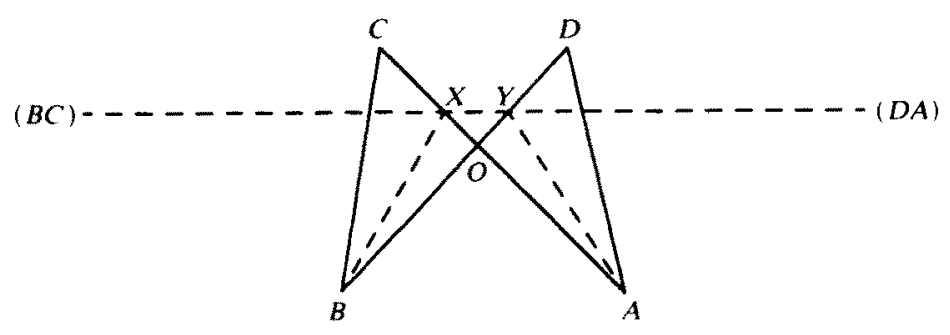

Fig. 8. $\measuredangle B V C<\measuredangle B X C=120^{\circ}$.

Corollary 1. If $\Varangle C+\Varangle D \geq 240^{\circ}$, then the $A D-B C$ tree does not exist.

Proof. Without loss of generality assume $\Varangle D \geq 120^{\circ}$. Then

$$
\Varangle(B C) D A=\Varangle D-\Varangle C D(B C)>\not \subset D-\left\{180^{\circ}-(\Varangle C+60)\right\} \geq 120^{\circ} \text {. }
$$

Corollary 2. If $\Varangle C+\Varangle D \geq 300^{\circ}$, then the spanning tree consisting of the three edges $[D, A],[C, D]$ and $[B, C]$ is the $S M T$.

Proof. From Corollary 1 the $A D-B C$ tree does not exist. Extend $[A, D]$ and $[B, C]$ to meet at $E$. Then $\measuredangle C+\not \subset D \geq 300^{\circ}$ implies that $\measuredangle E \geq 120^{\circ}$. Therefore $\Varangle B O A>\measuredangle E \geq 120^{\circ}$. Hence the $A B-C D$ tree does not exist. Nor can an SMT contain a single Steiner point since any three points of $\{A, B, C, D\}$ contain an angle of at least $120^{\circ}$. So the SMT must be a minimal spanning tree. Finally, it is easily verified that $d[A, B]>\max \{d[A, C], d[B, D]\}>$ $\max \{d[D, A], d[C, D], d[B, C]\}$.

\section{Can the Obtuse Full Steiner Tree Be an SMT?}

We give an example which answers the question posed in the heading in the affirmative.

Let $A B C D$ be a convex polygon such that $\Varangle A=\Varangle B>120^{\circ}, d[A, D]=d[B, C]$, $\measuredangle(C D) A B=\Varangle A B(C D)<120^{\circ}$ and $\measuredangle B O A=90^{\circ}$ (the existence of such a polygon is without question). By Theorem 6 and Corollary 1 the $A B-C D$ tree exists but not the $A D-B C$ tree. By Theorem 5 the $A B-C D$ tree is the SMT. It is clear that by a continuity argument we can increase $d[A, B]$ by a tiny amount such that $\Varangle B O A>90^{\circ}$ but nothing else is changed qualitatively. Hence the $A B-C D$ tree is the SMT.

Next we give some sufficient conditions under which the obtuse full Steiner tree can be ruled out as an SMT.

Theorem 7. Suppose that $\Varangle A \geq 120^{\circ}, \Varangle B \geq 120^{\circ}$. Construct $\left[A, D^{\prime}\right]$ and $\left[B, C^{\prime}\right]$ such that $\Varangle D^{\prime} A B=\Varangle A B C^{\prime}=120^{\circ}, d[A, D]=d\left[A, D^{\prime}\right]$ and $d[B, C]=d\left[B, C^{\prime}\right]$. Let $\left[A, C^{\prime}\right]$ and $\left[B, D^{\prime}\right]$ intersect at $O^{\prime}$. If $\Varangle B O^{\prime} A>90^{\circ}$, then the spanning tree consisting of the three edges $[A, D],[A, B]$, and $[B, C]$ is the $S M T$. 


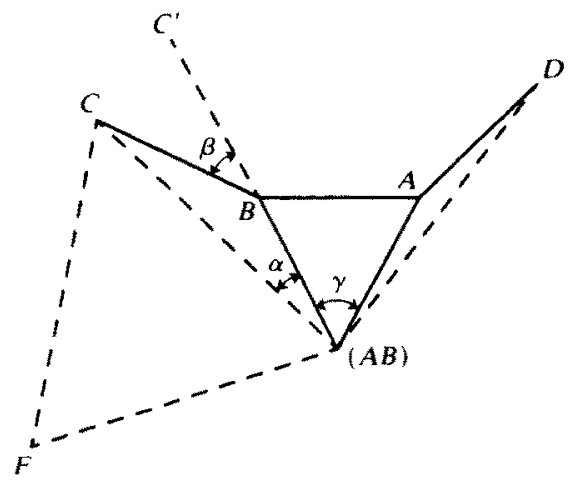

Fig. 9. The $d[(A B),(C D)]$ is minimized at $\beta=0^{\circ}$,

Proof. From Corollary 2 of Theorem 6 we need only consider the case $\Varangle A+\Varangle B<$ 300. Construct equilateral triangles $A B(A B)$ and $(A B) C F$. Define $\Varangle B(A B) C=\alpha$, $\Varangle C^{\prime} B C=\beta$, and $\triangle D(A B) B=\gamma$, where $0^{\circ} \leq \alpha<60^{\circ}, 0^{\circ} \leq \beta<180^{\circ}-\Varangle A$, and $60^{\circ} \leq$ $\gamma<90^{\circ}$ (see Fig. 9). We first show that for $\Varangle A \geq 120^{\circ}$ fixed, $d[D, F]$ achieves a minimum at $\beta=0$ :

$$
\begin{aligned}
d^{2}[D, F]= & d^{2}[D,(A B)]+d^{2}[C,(A B)] \\
& -2 d[D,(A B)] d[C,(A B)] \cos \left(\gamma+60^{\circ}+\alpha\right) \\
= & d^{2}[D,(A B)]+d^{2}[B, C]+d^{2}[B,(A B)]+2 d[B, C] d[B,(A B)] \cos \beta \\
& -2 d[D,(A B)] d[C,(A B)]\left\{\cos \left(\gamma+60^{\circ}\right) \cos \alpha-\sin \left(\gamma+60^{\circ}\right) \sin \alpha\right\}
\end{aligned}
$$

But

$$
\sin \alpha=d[B, C] \sin \beta / d[C,(A B)]
$$

and

$$
\cos \alpha=\left\{\frac{d^{2}[C,(A B)]-d^{2}[B, C] \sin ^{2} \beta}{d^{2}[C,(A B)]}\right\}^{1 / 2}=\frac{d[B, C] \cos \beta+d[B,(A B)]}{d[C,(A B)]}
$$

Therefore

$$
\begin{aligned}
d^{2}[D, F]= & d^{2}[D,(A B)]+d^{2}[B, C]+d^{2}[B,(A B)] \\
& +2 d[B, C]\left\{d[B,(A B)] \cos \beta-d[D,(A B)] \cos \left(\gamma+60^{\circ}+\beta\right)\right\} \\
& -2 d[D,(A B)] d[B,(A B)] \cos \left(\gamma+60^{\circ}\right)
\end{aligned}
$$

Define

$$
f(\beta)=d[B,(A B)] \cos \beta-d[D,(A B)] \cos \left(\gamma+60^{\circ}+\beta\right)
$$

Then

$$
f^{\prime}(\beta)=-d[B,(A B)] \sin \beta+d[D,(A B)] \sin \left(\gamma+60^{\circ}+\beta\right)
$$


Noting that $f(\beta)$ is a positive trigonometric function,

$$
f^{\prime \prime}(\beta)=-f(\beta)<0
$$

Hence $f(\beta)$ achieves its minimum at one of its two extreme values, i.e., $\beta=0^{\circ}$ or $\beta=180^{\circ}-\measuredangle A$.

But

$$
\begin{aligned}
& f\left(180^{\circ}-\Varangle A\right)-f\left(0^{\circ}\right) \\
&= d[B,(A B)](-\cos \Varangle A)+d[D,(A B)] \cos \left(\gamma+60^{\circ}-\not A\right) \\
&-d[B,(A B)]+d[D,(A B)] \cos \left(\gamma+60^{\circ}\right) \\
&=-d[B,(A B)](1+\cos \measuredangle A)+d[D,(A B)]\left\{\cos \left(\gamma+60^{\circ}-\not A\right)+\cos \left(\gamma+60^{\circ}\right)\right\} \\
&=-d[B,(A B)] 2 \cos ^{2} \frac{\not A}{2}+d[D,(A B)] 2 \cos \frac{2 \gamma+120^{\circ}-\Varangle A}{2} \cos \frac{\not A}{2} \\
&= 2 \cos \frac{\not A}{2}\left\{d[D,(A B)] \cos \frac{2 \gamma+120^{\circ}-\not A}{2}-d[B,(A B)] \cos \frac{\not A}{2}\right\}>0
\end{aligned}
$$

since

$$
d[D,(A B)]>d[B,(A B)]
$$

and

$$
90^{\circ}>\frac{\not A}{2}>\frac{2 \gamma+120^{\circ}-\not A}{2}>0
$$

implies

$$
\begin{gathered}
\cos \frac{\not A}{2}>0, \\
\cos \frac{2 \gamma+120^{\circ}-\not A A}{2}>\frac{\cos \measuredangle A}{2} .
\end{gathered}
$$

Similarly, we can show that for $\measuredangle B \geq 120^{\circ}$ fixed, $d[D, F]$ achieves a minimum when $D=D^{\prime}$. Combining the two arguments we conclude that $d[D, F] \geq$ $d\left[D^{\prime}, F^{\prime}\right]$, where $F^{\prime}=\left(C^{\prime},(A B)\right)$. Let $T$ be a Steiner tree for $\{A, B, C, D\}$ having at least one Steiner point. By Theorem $6 T$ is not the $A D-B C$ tree. Furthermore,

$$
\begin{aligned}
& \text { length of } T \geq d[D, F] \\
& \text { by Lemma } 5 \\
& \geq d\left[D^{\prime}, F^{\prime}\right]=d\left[(A B),\left(C^{\prime} D^{\prime}\right)\right] \\
& \geq d\left[\left(A D^{\prime}\right),\left(B C^{\prime}\right)\right] \\
& \text { by Lemma } 2 \\
& =d\left[A, D^{\prime}\right]+d[A, B]+d\left[B, C^{\prime}\right] \\
& =d[A, D]+d[A, B]+d[B, C] \text {. }
\end{aligned}
$$




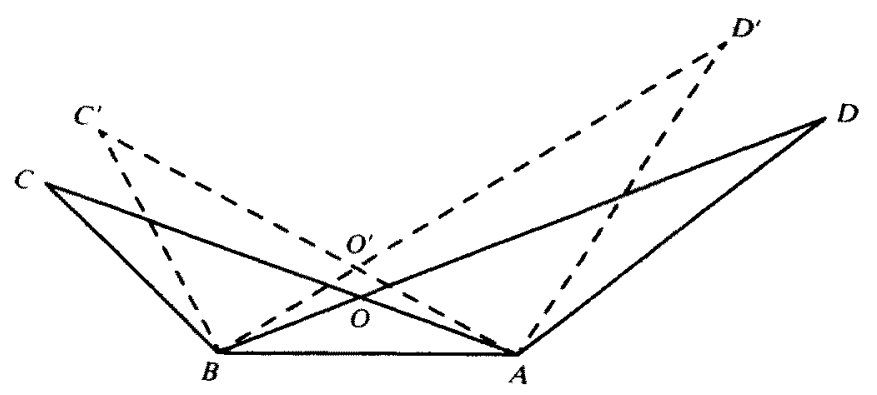

Fig. 10. $\measuredangle B O^{\prime} A-90^{\circ} \geq \Varangle B O A-\left(\measuredangle A+\Varangle B-150^{\circ}\right)$.

Hence the SMT for $\{A, B, C, D\}$ is the minimal spanning tree. But all spanning trees other than the one given in Theorem 7 contain an angle of less than $120^{\circ}$ and hence cannot be an SMT. The proof is complete.

Corollary. Suppose that $\Varangle A \geq 120^{\circ}, \Varangle B \geq 120^{\circ}$, and $\Varangle B O A>\Varangle A+\Varangle B-150^{\circ}$. Then the spanning tree given in Theorem 7 is the SMT (Fig. 10).

Proof. Note that

$$
\Varangle A D^{\prime} D=\Varangle D^{\prime} D A=90^{\circ}-\measuredangle D A D^{\prime} / 2>60^{\circ} .
$$

Hence $\Varangle A+\Varangle B D^{\prime} D>180^{\circ}$ in the quadrilateral $A B D^{\prime} D$. By Lemma $4 \Varangle D B D^{\prime} \leq$ $\Varangle D A D^{\prime}$. Similarly, we can prove $\Varangle C^{\prime} A C \leq \Varangle C^{\prime} B C$. It follows

$$
\begin{aligned}
\Varangle B O^{\prime} A-90^{\circ} & =\Varangle B O A-\Varangle D B D^{\prime}-\Varangle C^{\prime} A C-90^{\circ} \\
& \geq \Varangle B O A-\Varangle D A D^{\prime}-\Varangle C^{\prime} B C-90^{\circ} \\
& =\Varangle B O A-\left(\Varangle A+\Varangle B-150^{\circ}\right)
\end{aligned}
$$

Theorem 8. Suppose that $\Varangle A<120^{\circ}$ and $\Varangle(D A) B C \geq 120^{\circ}$. Construct $\left[B, C^{\prime}\right]$ such that $\chi(D A) B C^{\prime}=120^{\circ}$ and $d\left[B, C^{\prime}\right]=d[B, C]$. Let $\left[A, C^{\prime}\right]$ and $[B, D]$ intersect at $O^{\prime}$. If ${ }_{4} B O^{\prime} A>90^{\circ}$, then an $S M T$ is either $T_{A}$ or $T_{C}$.

Proof. It is easily verified that every spanning tree contains an angle of less than $120^{\circ}$ and hence cannot be an SMT. Furthermore, $T_{B}$ and $T_{D}$ do not exist by angle considerations, and the $A D-B C$ tree does not exist by Theorem 6 . Hence, if the $A B-C D$ tree does not exist, then Theorem 8 is trivially true. Therefore we may assume that the $A B-C D$ tree $T$ exists and intersects $\left[B, C^{\prime}\right]$ at $C^{*}$. In $\triangle C^{\prime} C^{*} C, \Varangle C^{\prime}=\Varangle B C C^{\prime} \geq \Varangle C$, hence $d\left[C, C^{*}\right] \geq d\left[C^{\prime}, C^{*}\right]$. Suppose that $C$ is adjacent to the Steiner point $s$ in $T$. Let $T^{\prime}$ be obtained from $T$ by substituting $\left[C^{\prime}, s\right]$ for $[C, s]$. Then

$$
d[C, s]=d\left[C, C^{*}\right]+d\left[C^{*}, s\right] \geq d\left[C^{*}, C^{*}\right]+d\left[C^{*}, s\right] \geq d\left[C^{\prime}, s\right] .
$$


Note that $T^{\prime}$ has the same topology as the $A B-C^{\prime} D$ tree. Hence

$$
\begin{aligned}
d[(A B),(C D)] & =\text { length of } T \\
& \geq \text { length of } T^{\prime} \\
& \geq d\left[(A B),\left(C^{\prime} D\right)\right] \\
& >d\left[\left(D A, B C^{\prime}\right)\right] \quad \text { by Lemma } 2 \\
& =\text { length of } T_{C^{\prime}} \\
& =\text { length of } T_{C} .
\end{aligned}
$$

The proof is complete.

Corollary. Suppose that $\Varangle A<120^{\circ}, \quad \Varangle(D A) B C \geq 120^{\circ}$, and $\Varangle B O A \geq$ $\measuredangle(D A) B C-30^{\circ}$. Then an $S M T$ is either $T_{A}$ or $T_{C}$.

Proof.

$$
\begin{aligned}
\Varangle B O^{\prime} A-90^{\circ} & =\Varangle B O A-\not C^{\prime} A C-90^{\circ} \\
& \geq \Varangle B O A-\Varangle C^{\prime} B C-90^{\circ} \\
& =\Varangle B O A-\left(\Varangle(D A) B C-120^{\circ}\right)-90^{\circ} \\
& =\Varangle B O A-\left(\Varangle(D A) B C-30^{\circ}\right) .
\end{aligned}
$$

\section{An Imbedding Property}

One may wonder why we want to study the properties of an SMT for three or four regular points since there are only a small number of topologies and one can construct all Steiner trees and compare them without too much difficulty. The merit of such study lies in the fact that Steiner trees for a large number of regular points can contain subtrees of three or four points and understanding the small trees can help us to understand the big trees. To make our results on small trees more useful it is desirable to state the properties in as broad a term as possible. The following theorem represents such an effort.

Theorem 9. Let $A B C D$ be a convex quadrilateral with $\Varangle A \geq 120^{\circ}, \Varangle B \geq 120^{\circ}$, and $\Varangle B O A \geq \measuredangle A+\measuredangle B-150^{\circ}$. Let $A^{\prime} B^{\prime} C^{\prime} D^{\prime}$ be a quadrilateral imbedded in $A B C D$ with $A^{\prime}, B^{\prime}$ on $[A, B]$ and $C^{\prime}, D^{\prime}$ on $[C, D]$. Then an SMT for $\left\{A^{\prime}, B^{\prime}, C^{\prime}, D^{\prime}\right\}$ cannot be full.

Proof. Let $\left[A^{\prime}, C^{\prime}\right]$ and $\left[B^{\prime}, D^{\prime}\right]$ meet at $O^{\prime}$. Then clearly, $\Varangle B^{\prime} O^{\prime} A \geq \Varangle B O A$. Note that $\Varangle A^{\prime}+\Varangle B^{\prime}=\Varangle A+\not B \geq 240^{\circ}$. By Corollary 1 of Theorem 6 the $A^{\prime} D^{\prime}-B^{\prime} C^{\prime}$ tree does not exist. Without loss of generality assume $\Varangle B^{\prime} \geq 120^{\circ}$. If $4 A^{\prime} \geq 120^{\circ}$ also, then by Theorem 7 the $A^{\prime} B^{\prime}-C^{\prime} D^{\prime}$ tree is not an SMT. Therefore assume $\Varangle A^{\prime}<120^{\circ}$. We now show that $\Varangle\left(D^{\prime} A^{\prime}\right) B^{\prime} C^{\prime} \geq 120^{\circ}$ and $\Varangle B^{\prime} O^{\prime} A^{\prime}>\Varangle\left(A^{\prime} D^{\prime}\right) B^{\prime} C^{\prime}-30^{\circ} \quad$ (Fig. 11). $\Varangle\left(D^{\prime} A^{\prime}\right) B^{\prime} C^{\prime}=\Varangle B-\Varangle A B\left(D^{\prime} A^{\prime}\right)=$ $\Varangle B-\left(180^{\circ}-\not A^{\prime}-60^{\circ}-\triangle B\left(D^{\prime} A^{\prime}\right) A\right)>\measuredangle A+\not B-120^{\circ}=120^{\circ}$. 


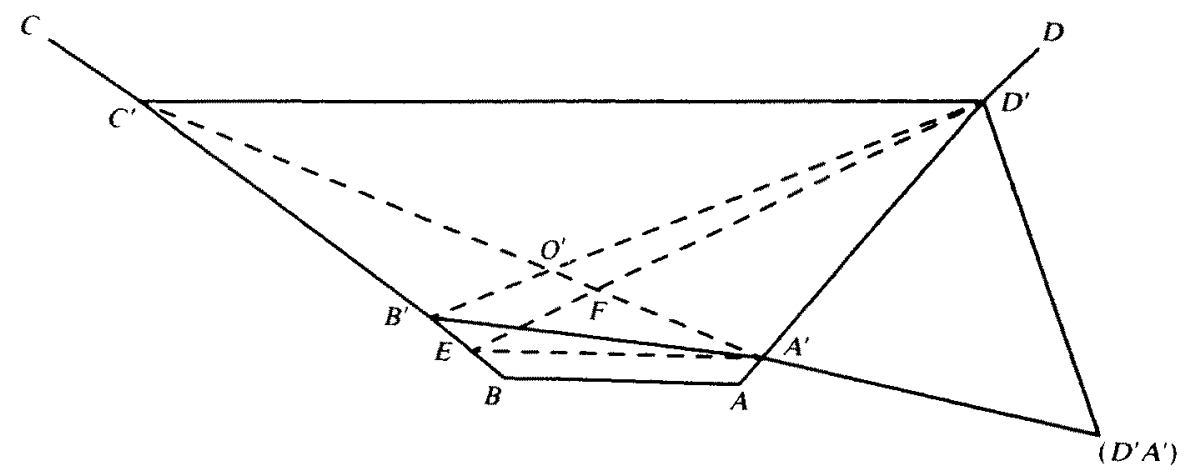

Fig. 11. $\varangle B^{\prime} O^{\prime} A>\measuredangle G^{\prime} B^{\prime} C^{\prime}-30^{\circ}$.

Let $E$ be a point on $[B, C]$ such that $\left[A^{\prime}, E\right]$ is parallel to $[A, B]$. Let $\left[D^{\prime}, E\right]$ cross $\left[A^{\prime}, C^{\prime}\right]$ at $F$. Then

$$
\Varangle E F A^{\prime}>\measuredangle B O A \geq \Varangle A+\not B B-150^{\circ}=\Varangle D^{\prime} A^{\prime} B^{\prime}+\not \Varangle A^{\prime} B^{\prime} C^{\prime}-150^{\circ} \text {. }
$$

Since

$$
\Varangle\left(D^{\prime} A^{\prime}\right) A^{\prime} E=60^{\circ}+\Varangle D^{\prime} A^{\prime} E \geq 180>\measuredangle\left(D^{\prime} A^{\prime}\right) A^{\prime} B^{\prime}
$$

we have

$$
\begin{aligned}
\Varangle B^{\prime}\left(D^{\prime} A^{\prime}\right) A^{\prime} & \leq \Varangle B^{\prime}\left(D^{\prime} A^{\prime}\right) E & & \text { since } \Varangle A \geq 120^{\circ} \\
& <\Varangle B^{\prime} D^{\prime} E & & \text { by Lemma } 4 .
\end{aligned}
$$

Hence

$$
\begin{aligned}
\not B^{\prime} O^{\prime} A^{\prime} & =\Varangle D^{\prime} F O^{\prime}+\Varangle O^{\prime} D^{\prime} F \\
& =\Varangle E F A^{\prime}+\Varangle B^{\prime} D^{\prime} E \\
& >\Varangle D^{\prime} A^{\prime} B^{\prime}+\Varangle A^{\prime} B^{\prime} C^{\prime}-150^{\circ}+\Varangle B^{\prime}\left(D^{\prime} A^{\prime}\right) A^{\prime} \\
& =\Varangle\left(D^{\prime} A^{\prime}\right) A^{\prime} B^{\prime}-60^{\circ}+\Varangle A^{\prime} B^{\prime} C^{\prime}-150^{\circ}+\Varangle B^{\prime}\left(D^{\prime} A^{\prime}\right) A^{\prime} \\
& =180^{\circ}-\Varangle A^{\prime} B^{\prime}\left(D^{\prime} A^{\prime}\right)+\Varangle A^{\prime} B^{\prime} C^{\prime}-210^{\circ} \\
& =\Varangle\left(D^{\prime} A^{\prime}\right) B^{\prime} C^{\prime}-30^{\circ} .
\end{aligned}
$$

By the corollary of Theorem 8 an SMT on the four points $\left\{A^{\prime}, B^{\prime}, C^{\prime}, D^{\prime}\right\}$ cannot be full.

\section{References}

1. F. R. K. Chung and R. L. Graham, A new bound for euclidean Steiner minimal trees, Ann. N.Y. Acad. Sci. 440 (1985), 328-346.

2. D. Z. Du, F. K. Hwang, and J. F. Weng, Steiner minimal trees on zigzag lines, Trans. Amer. Math. Soc. 278 (1983), 149-156. 
3. D. Z. Du and F. K. Hwang, Steiner minimal trees for bar waves, to appear.

4. M. R. Garey, R. L. Graham, and D. S. Johnson, The complexity of computing Steiner minimal tress, SIAM J. Appl. Math. 32 (1977), 835-859.

5. E. N. Gilbert and H. O. Pollak, Steiner minimal trees, SIAM J. Appl. Math. 16 (1968), 1-29.

6. Z. A. Melzak, On the problem of Steiner, Canad. Math. Bull. 4 (1960), 143-148.

7. Dame K. Ollerenshaw, Minimum networks linking four points in a plane, Inst. Math. Appl. 15 (1978), 208-211.

8. H. O. Pollak, Some remarks on the Steiner problem, J. Combin. Theor. Ser. A 24 (1978), 278-295.

Received January 10, 1986. 